\title{
Level of Prostate Cancer Diagnosis Using MRI-TRUS Fusion Biopsy in Patients with a Negative History of TRUS Biopsy
}

\author{
Alireza Lashey ${ }^{1}$, Said Yaghoob Sehri ${ }^{1}$, Ahmad Aghai Meibodi ${ }^{1}$, Mahyar Ghafari ${ }^{2}$, Jafar Gholivandan ${ }^{1}$, \\ Mohamad Solymani ${ }^{1}$ and Mahmood Bakhtiyari ${ }^{3,{ }^{*}}$ \\ ${ }^{1}$ Department of Urology, Shahid Beheshti University of Medical Sciences, Tehran, Iran \\ ${ }^{2}$ Department of Radiology, Shahid Beheshti University of Medical Sciences, Tehran, Iran \\ ${ }^{3}$ Non-Communicable Diseases Reseach Center, Alborz University of Medical Sciences, Karaj, Iran \\ "Corresponding author: Non-Communicable Diseases Reseach Center, Alborz University of Medical Sciences, Karaj, Iran. Email: mahmood.bakhtiyari@ymail.com
}

Received 2019 May 13; Accepted 2019 June 10.

\begin{abstract}
Background: Given the popularity of prostate specific antigen (PSA) testing in recent years, the number of patients undergoing diagnostic prostate biopsy has increased. The transrectal ultrasound-guided (TRUS) biopsy is considered as the gold standard for prostate cancer detection, although has a low sensitivity.

Objectives: The current study aimed at enhancing prostate cancer diagnosis using MRI-TRUS fusion biopsy in patients with negative history of TRUS biopsy.

Methods: In the current study, patients undergone TRUS prostate biopsy with benign results that were candidates for repeat biopsy were recruited. After making the preparations, patients underwent magnetic resonance imaging (MRI)-TRUS fusion biopsy. Gleason score, the number of involved cores, perineural invasion, perilymphovascular invasion, and the percentage of core involvement were recorded.

Results: Of the 191 patients, 70 (36.6\%) had positive biopsies. The frequency of non-detectable cancers by targeted biopsy based on the level of cancer risk showed that at the very high-risk level, five (29.4\%) and at high-risk level, two (11.7\%) subjects were not recognized. The mean Gleason score in targeted $(7.47 \pm 0.99)$ and random $(7.13 \pm 1.04)$ positive biopsies showed a significant difference between the two groups $(\mathrm{P}=0.045)$. Targeted biopsies are better than random ones to detect high-risk $(33.9 \%$ vs $29.2 \%, \mathrm{P}=0.013$ respectively) and very high-risk cancers ( $45.3 \%$ vs. $41.5 \%, \mathrm{P}=0.05$ respectively).

Conclusions: The combination of both biopsy approaches is suggested to offer a reliable method with high rate of tumor detection.
\end{abstract}

Keywords: Prostate Cancer, MRI-TRUS, TRUS Fusion Biopsy, Biopsy

\section{Background}

It is estimated that 1.3 million new cases of prostate cancer occurred worldwide, resulting in 359,000 related deaths in 2018. Prostate malignant tumor is the 2 nd most frequent cancer in males and the 5 th highest mortality rate among the common cancers in them (1).

Given the popularity of prostate specific antigen (PSA) testing in recent years, the number of patients undergoing diagnostic prostate biopsy has increased (2). The transrectal ultrasound guided (TRUS) biopsy is considered as the gold standard for prostate cancer detection, although has a low sensitivity (39\% -52\%). Physicians increasingly encounter patients with clinical signs of prostate cancer, but negative TRUS-guided biopsy results. This method is associated with the underdetection of high-grade prostate cancers and the overdetection of low-grade cancers (3-5). Re- cently, magnetic resonance imaging (MRI)-guided biopsy is used in suspected cases with prior negative biopsy for the diagnosis of prostate cancer, especially its significant types (6).

In recent years, urologists increasingly use MRI-TRUS fusion biopsy to detect prostate cancer (7). This technique was designed to diagnose suspicious cases identified on MRI (8). In MRI-TRUS fusion biopsy, the prostate is imaged using ultrasound, as it is performed in recent decades; but if any suspicious matter occurs, the MRI of that prostate implemented earlier and stored in the device, is fused with real-time ultrasound using a digital connection. The advantage of MRI-TRUS fusion biopsy is its potential to be used in outpatient clinics just in a few minutes under local anesthesia as well as high reliability and accuracy in prostate cancer diagnosis $(9,10)$. 


\section{Objectives}

The current study aimed at evaluating the detection rate of MRI-TRUS fusion biopsy in prostate cancer in cases with prior negative biopsy.

\section{Methods}

\subsection{Study Sample}

In a historical cohort study, all patients who were referred from 1 June 2017 to 30 April 2018 to Modarres, Rasoul Akram, and Payambaran hospitals in Tehran, Iran that undergone transrectal ultrasound biopsy of the prostate, and their benign pathology reports were based on the following criteria were enrolled: (1) increased PSA, (2) lack of PSA compared to previous biopsy, (3) suspicious digital rectal exam, (4) atipical small acinar proliferation in the previous pathology, (5) atypical gland suspicious of cancer in the previous pathology, (6) high-grade prostatic intraepithelial neoplasia (PIN), at least two cores in the previous pathology, and (7) intraductal carcinoma undergoing biopsy in the previous pathology. In the present study, all patients with febrile illnesses, patients with uncorrected coagulation disorders, the ones suspected of metastatic prostate cancer, and also those who referred for the first biopsy were excluded.

\subsection{Data Collection}

Patients referred to Modares, Rasoul Akram, and Payambaran hospitals were asked to sign the written consent form and complete the basic demographic information questionnaire and then, their prostate volume was determined by a radiologist. Other basic and clinical information of patients including age, prostate volume, total and free PSA level, PSA density, rectal examination findings, number of random and targeted biopsies, biopsy area, Gleason score, and core conflict percentage were recorded in data collection forms. All patients were then subjected to MP-MRI and MR images were interpreted by a radiologist with prostate MRI experience that was blind to research hypothesis. The quality of the completed forms was controlled continuously in order to ensure the accuracy of the information recorded. In the first phase, a number of completed forms were randomly selected and evaluated by another radiologist. At the end of the data collection, the forms were independently logged and coded by two individuals into Excel software. In the case of discrepancies, review of the questionnaire, completion of the contact form, or exclusion of the subject from the study was considered.

\subsection{Procedures and Definitions of Variables}

The type of visible lesions in the prostate is classified into five groups based on the prostate imaging reporting and data system (PIRADS) version 2 as very low (PIRADS 1), low (PIRADS 2), moderate (PIRADS 3), high (PIRADS 4), and very high risk of malignancy (PIRADS 5). Subsequently, patients underwent MRI-TRUS fusion biopsy. The patients were visited by the cardiologist and in case of using aspirin or antiplatelet and anti-coagulant drugs, their regimens were ceased or changed. Biopsy was not performed febrile subjects or the ones suspected of coagulopathy (based on coagulation tests). Oral ofloxacin $300 \mathrm{mg}$ and metronidazole $500 \mathrm{mg}$ were prescribed twice daily for prophylaxis from 24 hours before to five days after biopsy. One hour before biopsy, $500 \mathrm{mg}$ amikacin immediately before and 500 $\mathrm{mg}$ lefloxacin were intravenously injected. Biopsy was performed in left lateral decubitus with local anesthesia $(2 \%$ lidocaine injection in the prostate capsule) using $18 \mathrm{G}$ calibre needles.

According to the number of suspected lesions in the MRI report and based on the prostate volume, 12 to $36 \mathrm{pts}$ were taken from each patient. After the biopsy, the patient was monitored for an hour and then the possible complications including urinary retention, fever and chills, hematuria and rectal cancer were explained to the patient, and it was advised to contact the emergency department immediately if any of the side effects occurred. The specimens were sent to the pathology laboratory and based on the obtained results, information such as Gleason score, number of involved cores, perineural invasion, perilymphaticvascular invasion, and the percentage of core involvement were recorded in the information form of each patient. The risk of prostate cancer was classified into four groups: PSA risk $<10 \%$, Gleason score 2 to 6 , and unilateral or less than $50 \%$ involvement of each core as low risk, PSA risk $<10 \%$, Gleason score 3 - 4, and bilateral involvement of each core as moderate risk, Gleason score 3 - 4, PSA risk 10\% - 20\%, involvement of more than $50 \%$ of each core, and perineural invasion as high risk, and Gleason score $8-10$, PSA $>20 \%$, or perilymphatic-vascular invasion as very high risk. Also, PIRADS scores $\geq 3$ based on multiparametric (MP)-MRI findings were considered as abnormal and vice versa.

\subsection{Statistical Analysis}

The mean and standard deviation (SD) were used to express quantitative variables, and the frequency and percentage to compare the categorical variables. Independent $t$-test was used to compare the mean of quantitative variables between the two groups in case of normal distribution and otherwise, the Mann-Whitney test was employed. Chi-square test was used to evaluate the independence of 
variables between the two groups. To specify the shape of association between the variables, a fractional polynomial model was used. The ROC (receiver operating characteristic) logistic regression model was used to determine the area under the curve (AUC). All analyzes were performed using the STATA 13MP software.

\section{Results}

In the study, 191 patients referring to Modarres, Rasoul Akram, and Payambaran hospitals in Tehran were evaluated. The mean age of the subjects was $63.8 \pm 8.1$ years. The average prostate volume in the studied patients was $60.7 \pm$ $29.8 \mathrm{~mL}$. The results showed a positive correlation between prostate cancer $(\mathrm{PCa})$ and SPA density (Spearman's rho $=$ 0.29 , $\mathrm{P}$ value $=0.0001$ ). Furthermore, there was a negative significant correlation between the PCa and prostate volume (Spearman's rho $=-0.27$, $\mathrm{P}$ value $=0.0001$ ). The shape of association was showed using fracpoly model in Figure 1.

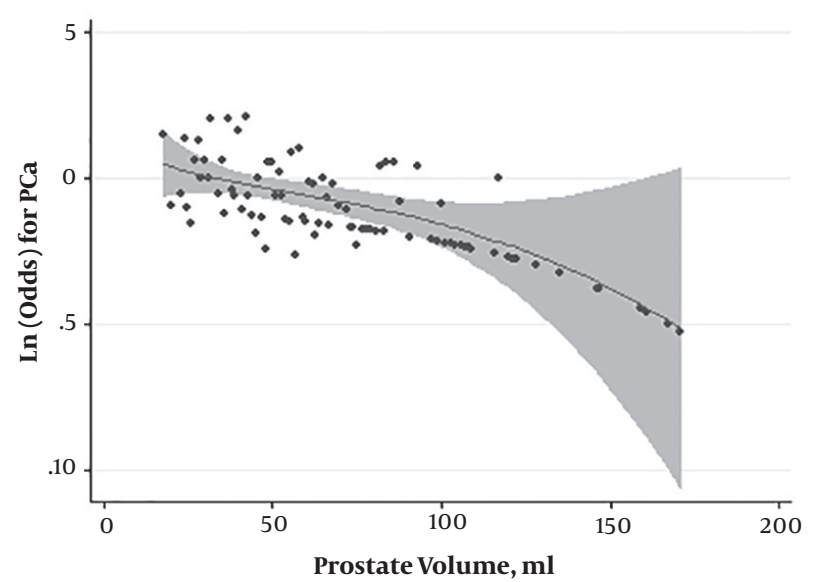

Figure 1. Shape of association between the PCa and prostate volume

A total of 70 (36.6\%) cases of positive biopsy were reported from 191 studied patients. Also, the highest rate of prostate cancer was observed in the age group of $65-74$ years (42.9\%). Out of 191 cases of targeted biopsy, 53 (27.75\%) were positive, while the number of positive cases for random biopsy was $63(34 \%)$. In terms of number, the targeted and random biopsies were $4.58 \pm 2.2$ and $9.35 \pm 1.76$, respectively; the overall number of random biopsies was almost double than the targeted ones (Table 1 ).

In the current study, the prevalence of prostate cancer in patients with and without lesion on MRI was $38.7 \%$ and $21.7 \%$, respectively (Table 2 ).
Table 1. Comparison of Positive and Negative Biopsy Results Based on Normal and Abnormal MR Images

\begin{tabular}{lccc}
\hline Variable & $\begin{array}{c}\text { Normal MRI, } \\
\text { No. (\%) }\end{array}$ & $\begin{array}{c}\text { Abnormal MRI, } \\
\text { No. (\%) }\end{array}$ & P Value \\
\hline Positive biopsy & $5(7)$ & $65(92.8)$ & 0.011 \\
\hline Negative biopsy & $18(14.9)$ & $103(85.1)$ & \\
\hline
\end{tabular}

Table 2. The Prostate Cancer Risk Stratification Based on Normal and Abnormal MR Images

\begin{tabular}{lccc}
\hline Grade & $\begin{array}{c}\text { Normal MRI, } \\
\text { No. (\%) }\end{array}$ & $\begin{array}{c}\text { Non-Normal } \\
\text { MRI, No. (\%) }\end{array}$ & P Value \\
\hline Low risk & $0(0)$ & $20(13)$ & 0.12 \\
\hline Intermediate risk & $2(40)$ & $6(9.2)$ & \\
\hline High risk & $2(40)$ & $18(27.7)$ & \\
\hline Very high risk & $1(20)$ & $28(43)$ & \\
\hline
\end{tabular}

The detection rate of significant cancer in the subjects with lesions on MRI was higher than that of the ones with normal MRI reports, although the difference was not statistically significant $(P=0.12)$. Figure 2 shows the frequency distribution of risk-based prostate cancer in different groups of PI-RADS score.

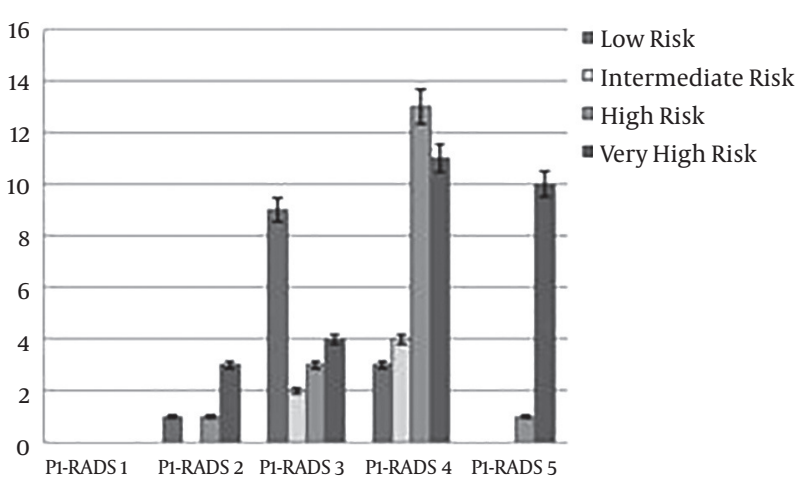

Figure 2. Frequency distribution of risk-based prostate cancer in different groups of PI-RADS score

The results showed that the rate of positive agreement between the positive random and targeted biopsies in the study was 33.5\%; a total of 48 subjects diagnosed with positive random biopsy were also positive for targeted biopsy.

The frequency of non-detectable cancers by targeted biopsy based on the level of cancer risk showed that at the very high risk level, five subjects (29.4\%) and at the high risk level, two subjects (11.7\%) were not recognized.

Also, the comparison of the mean Gleeson score in targeted $(7.47 \pm 0.99)$ and random $(7.13 \pm 1.04)$ positive biopsies showed a significant difference between the two groups $(\mathrm{P}=0.045)$. Table 3 shows the results of the compar- 
Table 3. Comparison of the Prevalence of Prostate Cancer in Terms of Positive Targeted and Random Biopsies

\begin{tabular}{lccc}
\hline Cancer Risk & $\begin{array}{c}\text { Positive Targeted } \\
\text { Biopsy, No. (\%) }\end{array}$ & $\begin{array}{c}\text { Positive Random } \\
\text { Biopsy, No. (\%) }\end{array}$ & P Value \\
\hline Low risk & $5(9.4)$ & $11(16.9)$ & 0.01 \\
$\begin{array}{l}\text { Intermediate } \\
\text { risk }\end{array}$ & $6(11.3)$ & $8(12.3)$ & 0.11 \\
\hline High risk & $18(33.9)$ & $19(29.2)$ & 0.013 \\
\hline Very high risk & $24(45.3)$ & $27(41.5)$ & 0.05 \\
\hline Total & $53(100)$ & $65(100)$ & - \\
\hline
\end{tabular}

ison of the prevalence of prostate cancer in terms of risk in the positive outcomes of the targeted and random biopsy.

The results shown on Table 3 indicate that targeted biopsies could better detect high and very high risk cancers compared with the random ones. Table 4 shows the potency of random and targeted biopsies to detect prostate cancer.

Data shown on Table 3 indicate that the potency of targeted biopsies to detect prostate cancer was higher than random biopsies, so that about $82 \%(n=24)$ of the total 29 cases were positive biopsy with a sensitivity of $75 \%-100 \%$ (Table 4). However, this amount was $6.8 \%$ for random biopsies.

The comparison of the sensitivity of targeted and random biopsy based on different prostate regions is shown in Table 5.

The results of the ROC regression model analysis showed that the AUC for targeted and random biopsies was $0.88 \%(0.83 \%)$ and $87.4 \%$ (99.9\% - 0.93\%), respectively (Figure 3).

The results of above-mentioned analyses showed that the sensitivity and negative predictive value for targeted and random biopsies were 75.7\% (64\% - 83\%), 87.7\% (81\% $94 \%)$, and $92.8 \%$ ( $88 \%$ - 97\%), 96\% (93\% - 99\%) respectively.

\section{Discussion}

In the current study, the PSA density, PI-RADS score, presence of lesion on MRI, and smaller prostate volume were correlated with a higher prevalence of prostate cancer.

In the present study, the frequency of prostate cancer diagnosis was $21.7 \%$ in cases with normal MR images and $38.7 \%$ in those with lesions visible on MRI. In subjects with abnormal MR images, the prevalence of prostate cancer at PI-RADS 4 and 5 was higher than PI-RADS1, 2, and 3 (64.6\%). Mean PI-RADS score was 3.7384 and 2.8058 in the subjects with positive and negative biopsies.

The diagnosis rate of prostate cancer in the current study had a significant and negative correlation with
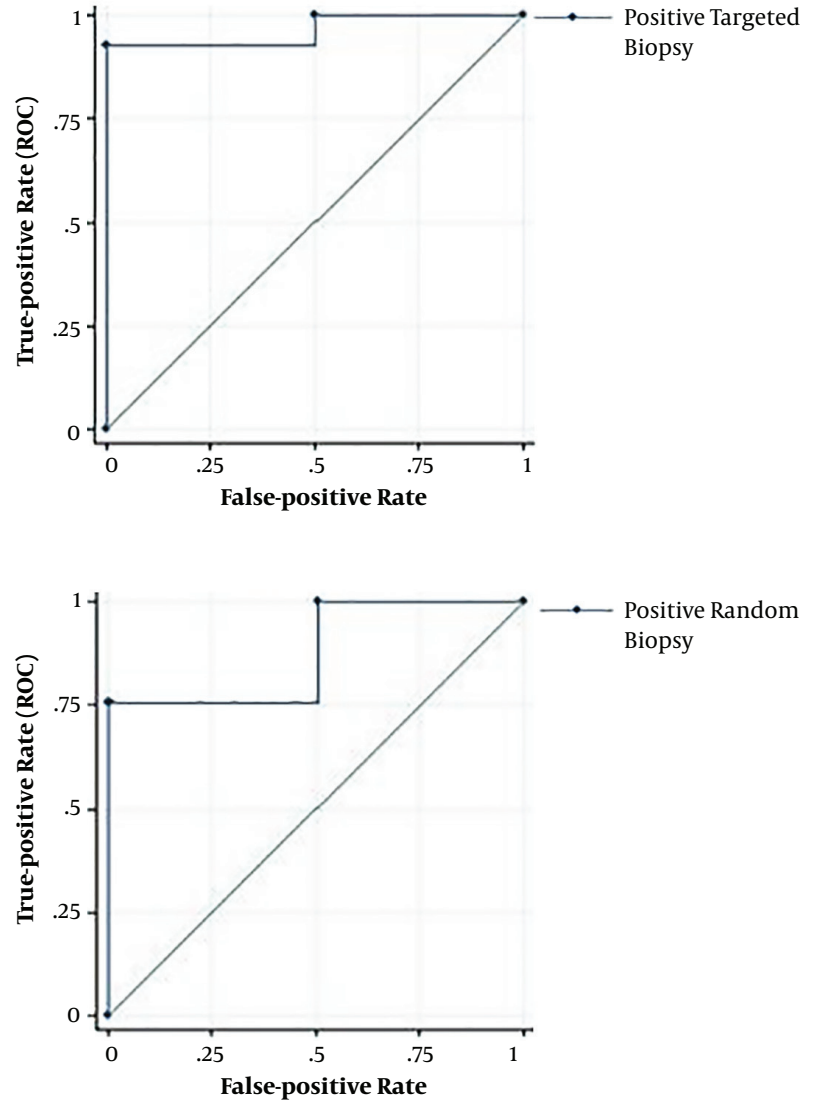

Figure 3. Targeted and random biopsy area under curve in studied participants

prostate volume (mean $66.8 \mathrm{~mL}$ in negative and $49.94 \mathrm{~mL}$ in positive biopsies). In studies by Al-Azab et al. (11), a smaller prostate volume was a predictive factor for the diagnosis of prostate cancer in TRUS biopsy. In the study by Regis et al. (12), on 175 patients with a prior negative TRUS biopsy, the rate of prostate cancer diagnosis was 33.1\% ( $\mathrm{n}=$ 58).

Furthermore, the presence of lesions on prostate MRI and higher PI-RADS score were correlated with a higher risk of significant prostate cancer. Kesch et al. (13), showed that in case of doubtful MRI lesions, applying the both biopsy methods offers maximum sensitivity for tumor detection.

Several methods are proposed for targeted biopsies (TBX) of mpMRI for suspicious core (14). Direct in-bore TBx can precisely address tumor site with direct image validation of needle placement within the target. Therefore, it can be considered as the gold standard of MRI-guided TBX, although it is costly, has lower access, and is not allowed for simultaneous systematic sampling. However, fusing mpMRI data with TRUS (MRI/TRUS fusion) pools, the su- 


\begin{tabular}{lccccc}
\hline Table 4. The Sensitivity of Random and Targeted Biopsies in Detecting Prostate Cancer Based on the Risk Groups \\
\hline \multirow{2}{*}{ Cancer Risk } & \multicolumn{3}{c}{ Sensitivity (\%) } & Total \\
\cline { 2 - 6 } & $\mathbf{0 - 2 5}$ & $\mathbf{2 5 - 5 0}$ & $\mathbf{5 0 - 7 5}$ & $\mathbf{7 5 - 1 0 0}$ & \\
\hline Targeted biopsy & & & & & $13(100)$ \\
\hline Low risk & $8(61.5)$ & $3(23)$ & $1(7.6)$ & $1(7.6)$ & $8(100)$ \\
\hline Intermediate risk & $2(25)$ & $0(0)$ & $0(0)$ & $6(75)$ & $20(100)$ \\
\hline High risk & $2(10)$ & $2(10)$ & $0(0)$ & $16(80)$ & $29(100)$ \\
\hline Very high risk & $5(17.3)$ & $0(0)$ & $0(0)$ & $24(82)$ & $70(100)$ \\
\hline Total & $17(24.3)$ & $5(7.2)$ & $1(1.4)$ & $47(67.1)$ & $13(100)$ \\
\hline Random biopsy & & & & & $8(100)$ \\
\hline Low risk & $13(100)$ & $0(0)$ & $0(0)$ & $0(0)$ & $20(100)$ \\
\hline Intermediate risk & $6(75)$ & $2(25)$ & $0(0)$ & $0(0)$ & $29(100)$ \\
\hline High risk & $9(45)$ & $6(30)$ & $3(15)$ & $2(10)$ & $70(100)$ \\
\hline Very high risk & $13(44.8)$ & $8(27.5)$ & $6(20.6)$ & $2(6.8)$ & $4(5.8)$ \\
\hline Total & $41(58.5)$ & $16(22.8)$ & $9(12.9)$ & & \\
\hline
\end{tabular}

\begin{tabular}{|c|c|c|c|c|}
\hline Positive Biopsy Rate & Apex & Mid & Base & Inner Lobe \\
\hline Random biopsy, \% & 27.2 & 33.6 & 25 & 33.3 \\
\hline Targeted biopsy, \% & 90 & 83.7 & 94.4 & 48 \\
\hline Pvalue & 0.001 & 0.005 & 0.001 & 0.01 \\
\hline
\end{tabular}

perior imaging of mpMRI coupled with the easier-to-use ultrasound guidance, which allows trained operators to complete TBx in real-time in an outpatient clinic, saves time and costs while preserving acceptable targeting accu$\operatorname{racy}(15,16)$.

The negative predictive value was $87.7 \%$ and $96 \%$ for targeted and random biopsies in the current study, respectively. In the study by Regis et al. on 175 patients with prior negative biopsy, MRI scans had a negative predictive value of $90.2 \%$ for not performing a biopsy on patients with a low risk of prostate cancer, which is somewhat consistent with the results of the present study.

The sensitivity of targeted biopsies was $75.7 \%$ in the diagnosis of prostate cancer, and $84.3 \%$ for significant cancers. On the contrary, in the study by Ahmed on 576 patients, the sensitivity of MP-MRI was 93\% in diagnosing significant prostate cancer (17). In the present study, the sensitivity of targeted biopsy to diagnose very high-risk and high-risk cancers was $45.3 \%$ and $33.9 \%$, respectively. The sensitivity of random biopsy to diagnose very high-risk and high-risk cancers was $41.5 \%$ and $29.3 \%$, respectively.

The difference in the diagnosis of very-high-risk and high-risk cancers in two groups was $8.5 \%$, in favor of targeted biopsy; the difference was reported 30\% in a study by Siddiqui et al. (18). In the present study, the sensitivity of targeted biopsy to diagnose low-risk cancer was 9.4\%, while it was $16.9 \%$ in random biopsy; the difference between the results was significant (7.5\%). In the study by Pokorny et al. (19), the sensitivity was $6.1 \%$ and $37.3 \%$ for the targeted and random biopsies, respectively. The important point was that in both of the aforementioned studies, most patients were the first-time biopsy cases.

In the present study, of the 70 cases diagnosed with prostate cancer, targeted biopsy failed to diagnose 17 cases (a false negative biopsy), of which nine cases (53\%) had a significant cancer. The results demonstrated the necessity of performing systematic random biopsy simultaneously with targeted biopsy.

In the present study, the sensitivity of targeted biopsy was $94.4 \%$ and $90 \%$ in the diagnosis of prostate cancer at the base and apex, respectively; whereas random biopsy had a sensitivity of $25 \%$ and $27.2 \%$ in the same regions, respectively. In the study by Sazuka et al. (20), results of permanent radical prostatectomy pathology of 158 patients were compared with those of pre-operative TRUS biopsy. In radical prostatectomy, $85 \%$ of cancer cases showed the apex involvement. Of 118 patients with apex involvement in permanent pathology, TRUS biopsy reported 53 cases (45\%) as negative. It demonstrated the advantage of MRI-guided targeted biopsy to diagnose prostate cancer in the apex, 
which is the most common region for positive margin in radical prostatectomy and where the TRUS biopsy has a high risk of missing.

In the present study, systematic random biopsy diagnosed more cases of prostate cancer (in total and based on risk classification) compared to that of targeted biopsy ( $\mathrm{n}$ $=65$ vs. $n=53$ ). Although this difference was not significant, based on the bias resulting from the larger number of random biopsies, multivariate analysis was performed and the sensitivity of biopsy methods was assessed based on the percentage of positive biopsies divided by the total number of biopsies. The sensitivity of targeted biopsy was significantly more than that of random biopsy in the general diagnosis of cancer and also in cancers with a higher relapse rate.

In the current study, although the diagnosis rate of prostate cancer was higher using random biopsy compared to targeted biopsy, the difference was not statistically significant; given to the percentage of positive biopsies divided by the total number of targeted biopsies, the sensitivity of targeted biopsy in the diagnosis of cancer, especially its significant type, was significantly higher than random method.

The sensitivity of targeted biopsy was significantly higher than random biopsy in the diagnosis of prostate cancer in the apex and base of the prostate. In addition to the abovementioned issues, MRI-guided in-bore prostate biopsies are linked with lower amounts of overall problems compared with TRUS-guided PB, comprising bleeding, hematuria, and hematospermia (21-23).

\subsection{Conclusions}

Most high-risk lesions identified with targeted and random biopsies were also positive for low-risk measures, and the number of cancer cases undiagnosed by targeted biopsy was high (24.2\%); however, 53\% of them had significant cancer, indicating the importance of performing random and targeted biopsies, simultaneously.

\section{Acknowledgments}

The authors would like to thank the Clinical Research Development Unit (CRDU) of Emam Ali Hospital, Alborz University of Medical Sciences, Karaj, Iran for their support, cooperation and assistance throughout the period of study.

\section{Footnotes}

Authors' Contribution: Study concept and design: Alireza Lashey, and Mahmood Bakhtiyari. Analysis and in- terpretation of data: Said Yaghoob Sehri, Mohamad Solymani and Mahyar Ghafari. Drafting of the manuscript: Mahmood Bakhtiyari. Critical revision of the manuscript for important intellectual content: Alireza Lashey, Mahyar Ghafari, Mohamad Solymani, Said Yaghoob Sehri, Jafar Gholivandan, Ahmad Aghai Meibodi. Statistical analysis: Ahmad Aghai Meibodi and Mahmood Bakhtiyari.

Conflict of Interests: The authors declared no conflict of interest.

Ethical Approval: The Ethics Committee of Shahid Beheshti University of Medical Sciences approved the proposal of this study. All the study procedures involving human participants were in accordance with the ethical standards of the institutional and/or national research committee as well as the Declaration of Helsinki, 1964 and its later revision or comparable ethical standards.

Funding/Support: There was no financial support for the study.

Patient Consent: Informed consent was obtained from all participants.

\section{References}

1. Bray F, Ferlay J, Soerjomataram I, Siegel RL, Torre LA, Jemal A. Global cancer statistics 2018: GLOBOCAN estimates of incidence and mortality worldwide for 36 cancers in 185 countries. CA Cancer J Clin. 2018;68(6):394-424. doi:10.3322/caac.21492. [PubMed: 30207593].

2. Taneja SS. Optimizing prostate biopsy strategies for the diagnosis of prostate cancer. Rev Urol. 2003;5(3):149-55. [PubMed: 16985636]. [PubMed Central: PMC1473018].

3. Robles J. Review of MRI-TRUS fusion-guided biopsy as alternative diagnostic technique over TRUS. UBSJ. 2017;23.

4. Overduin CG, Futterer JJ, Barentsz JO. MRI-guided biopsy for prostate cancer detection: A systematic review of current clinical results. Curr Urol Rep. 2013;14(3):209-13. doi: 10.1007/s11934-013-0323-z. [PubMed: 23568624].

5. Kasivisvanathan V, Rannikko AS, Borghi M, Panebianco V, Mynderse LA, Vaarala MH, et al. MRI-targeted or standard biopsy for prostatecancer diagnosis. N Engl J Med. 2018;378(19):1767-77. doi: 10.1056/NEJMoa1801993. [PubMed: 29552975].

6. Baco E, Rud E, Eri LM, Moen G, Vlatkovic L, Svindland A, et al. A randomized controlled trial to assess and compare the outcomes of twocore prostate biopsy guided by fused magnetic resonance and transrectal ultrasound images and traditional 12-core systematic biopsy. EurUrol.2016;69(1):149-56. doi:10.1016/j.eururo.2015.03.041. [PubMed: 25862143].

7. Rayn K, Gold S, Hale GR, Baiocco J, Bloom J, Valera V, et al. Is BMI a risk factor for active surveillance progression in patients with prostate cancer diagnosed by MRI-TRUS fusion biopsy? J Clin Oncol. 2018;36(6_suppl):124. doi:10.1200/JCO.2018.36.6_suppl.124.

8. Turkbey B, Xu S, Kruecker J, Locklin J, Pang Y, Bernardo M, et al. Documenting the location of prostate biopsies with image fusion. BJU Int. 2011;107(1):53-7. doi: 10.1111/j.1464-410X.2010.09483.x. [PubMed: 20590543]. [PubMed Central: PMC3272674].

9. Marks L, Young S, Natarajan S. MRI-ultrasound fusion for guidance of targeted prostate biopsy. Curr Opin Urol. 2013;23(1):43-50. doi: 10.1097/MOU.ob013e32835ad3ee. [PubMed: 23138468]. [PubMed Central: PMC3581822]. 
10. Tewes S, Hueper K, Hartung D, Imkamp F, Herrmann TR, Weidemann J, et al. Targeted MRI/TRUS fusion-guided biopsy in men with previous prostate biopsies using a novel registration software and multiparametric MRI PI-RADS scores: first results. World J Urol. 2015;33(11):170714. doi: 10.1007/s00345-015-1525-4. [PubMed: 25774003].

11. Al-Azab R, Toi A, Lockwood G, Kulkarni GS, Fleshner N. Prostate volume is strongest predictor of cancer diagnosis at transrectal ultrasound-guided prostate biopsy with prostate-specific antigen values between 2.0 and $9.0 \mathrm{ng} / \mathrm{mL}$. Urology. 2007;69(1):103-7. doi: 10.1016/j.urology.2006.09.041. [PubMed:17270628].

12. Regis L, Servian P, Celma A, Lopez R, Roche S, Planas J, et al. Mp48-12 multiparametric MRI increases the efficiency of the standard 12-core trus-guided repeated biopsies. J Urol. 2015;193(4S). doi: 10.1016/j.juro.2015.02.1686.

13. Kesch C, Radtke JP, Distler F, Boxler S, Klein T, Huttenbrink C, et al. [Multiparametric MRI and MRI-TRUS fusion biopsy in patients with prior negative prostate biopsy]. Urologe A. 2016;55(8):1071-7. German. doi: 10.1007/s00120-016-0093-6. [PubMed: 27168038].

14. Wegelin O, van Melick HHE, Hooft L, Bosch J, Reitsma HB, Barentsz JO, et al. Comparing three different techniques for magnetic resonance imaging-targeted prostate biopsies: A systematic review of inbore versus magnetic resonance imaging-transrectal ultrasound fusion versus cognitive registration. Is there a preferred technique? Eur Urol. 2017;71(4):517-31. doi: 10.1016/j.eururo.2016.07.041. [PubMed: 27568655].

15. Valerio M, Donaldson I, Emberton M, Ehdaie B, Hadaschik BA Marks LS, et al. Detection of clinically significant prostate cancer using magnetic resonance imaging-ultrasound fusion targeted biopsy: A systematic review. Eur Urol. 2015;68(1):8-19. doi: 10.1016/j.eururo.2014.10.026. [PubMed: 25454618].

16. Sonn GA, Natarajan S, Margolis DJ, MacAiran M, Lieu P, Huang J, et al. Targeted biopsy in the detection of prostate cancer using an office based magnetic resonance ultrasound fusion device. J Urol. 2013;189(1):86-91. doi: 10.1016/j.juro.2012.08.095. [PubMed: 23158413] [PubMed Central: PMC3561472].
17. Ahmed HU, El-Shater Bosaily A, Brown LC, Gabe R, Kaplan R, Parmar MK, et al. Diagnostic accuracy of multi-parametric MRI and TRUS biopsy in prostate cancer (PROMIS): A paired validating confirmatory study. Lancet. 2017;389(10071):815-22. doi: 10.1016/S01406736(16)32401-1. [PubMed: 28110982].

18. Siddiqui MM, Rais-Bahrami S, Turkbey B, George AK, Rothwax J, Shakir $\mathrm{N}$, et al. Comparison of MR/ultrasound fusion-guided biopsy with ultrasound-guided biopsy for the diagnosis of prostate cancer. JAMA. 2015;313(4):390-7. doi: 10.1001/jama.2014.17942. [PubMed: 25626035]. [PubMed Central: PMC4572575].

19. Pokorny MR, de Rooij M, Duncan E, Schroder FH, Parkinson R, Barentsz JO, et al. Prospective study of diagnostic accuracy comparing prostate cancer detection by transrectal ultrasound-guided biopsy versus magnetic resonance (MR) imaging with subsequent MR-guided biopsy in men without previous prostate biopsies. Eur Urol. 2014;66(1):22-9. doi: 10.1016/j.eururo.2014.03.002. [PubMed: 24666839].

20. Sazuka T, Imamoto T, Namekawa T, Utsumi T, Yanagisawa M, Kawamura K, et al. Analysis of preoperative detection for apex prostate cancer by transrectal biopsy. Prostate Cancer. 2013;2013:705865. doi: 10.1155/2013/705865. [PubMed: 23533779]. [PubMed Central: PMC3595663].

21. Kam SC, Choi SM, Yoon S, Choi JH, Lee SH, Hwa JS, et al. Complications of transrectal ultrasound-guided prostate biopsy: Impact of prebiopsy enema. Korean J Urol. 2014;55(11):732-6. doi: 10.4111/kju.2014.55.11.732. [PubMed: 25405015]. [PubMed Central: PMC4231150].

22. Loeb S, Vellekoop A, Ahmed HU, Catto J, Emberton M, Nam R, et al. Systematic review of complications of prostate biopsy. Eur Urol. 2013;64(6):876-92. doi: 10.1016/j.eururo.2013.05.049. [PubMed: 23787356].

23. Borghesi M, Ahmed H, Nam R, Schaeffer E, Schiavina R, Taneja S, et al. Complications after systematic, random, and image-guided prostate biopsy. Eur Urol. 2017;71(3):353-65. doi: 10.1016/j.eururo.2016.08.004. [PubMed: 27543165]. 